\title{
Cierre percutáneo de comunicación interauricular tipo ostium secundum en paciente con nacimiento anómalo de la arteria circunfleja originada en el seno coronariano derecho con recorrido retroaórtico
}

\author{
Percutaneous occlusion of interauricular septal defect in a patient with \\ anomalous left circumflex artery
}

Lucas Mas' ${ }^{1}$ Cristian Calenta' ${ }^{1}$ Pedro Zangroniz' ${ }^{1}$ Alejandro Peirone², Claudio Bonini ${ }^{3}$

\section{RESUMEN}

La CIA es la cardiopatía congénita detectada con mayor frecuencia en adultos. El cierre percutáneo es una alternativa segura y eficaz. Presentamos un paciente con CIA tipo ostium secundum al cual le realizamos cierre percutáneo. El análisis con ETE intraprocedimiento y la coronariografía selectiva permitió diagnosticar el nacimiento anómalo de la arteria circunfleja desde el seno coronariano derecho con recorrido retroaórtico en relación al borde anterosuperior del defecto. Este hallazgo es una potencial contraindicación del implante ya que se puede comprometer el flujo de vaso retroaórtico. Guiados por ETE, angiografía y electrocardiografía, pudimos llevar a cabo la oclusión del defecto. El seguimiento alejado demostró la no afectación de la misma.

Palabras claves: comunicación interauricular; anomalías coronarias; cierre percutáneo

\begin{abstract}
ASD is the most frequently detected congenital heart disease in adult patients. Percutaneous closure is a safe and effective treatment option. We present a patient with an ostium secundum type atrial septal defect in whom we performed percutaneous closure. Intraprocedural TEE analysis and selective coronary angiography allowed the diagnosis of an anomalous origin of the circumflex coronary artery originated from the right coronary sinus with a retroaortic course in relationship with the anterosuperior rim of the defect. This finding is a potential contraindication for device implantation because it can compromise the flow of the retroaortic coronary vessel. Guided by TEE, angiography and electrocardiography, we were able to perform the percutaneous ASD closure properly. Long term follow up showed an uneventful course.
\end{abstract}

Revista Argentina de Cardioangiología Intervencionista 2014;5(3):213-216

\section{INTRODUCCIÓN}

Las anomalías congénitas de las arterias coronarias son infrecuentes. La incidencia es del 1.69\% de los estudios angiográficos. El nacimiento de la arteria circunfleja desde la arteria coronaria derecha o en el seno coronariano derecho con recorrido retroaórtico, tiene una incidencia del $0.67 \%$ de todas las anomalías coronarias. Por otra parte, la comunicación interauricular (CIA) es la cardiopatía congénita más frecuentemente observada en el adulto, con una prevalencia del $40 \%$ y con predominio en el sexo femenino. Durante las dos pri-

\footnotetext{
- Hospital Provincial del Centenario, Rosario. Argentina.

2. Hospital Privado de Córdoba, Córdoba. Argentina

3. Diagnóstico Médico Oroño. Rosario. Argentina.
}

$\triangle$ Correspondencia: Pedro Zangroniz Urquiza 3101. Rosario. Santa Fe. Argentina Fax.03414377208 pedrozangroniz@fibertel.com.ar

Conflictos de intereses: no existen.

Recibido: 26-7-2014 | Aceptado: 10-8-2014 meras décadas de la vida, los pacientes suelen estar asintomáticos. Luego de los 20 años, el 50\% tiene algunos síntomas y a partir de la sexta década, el $100 \%$ presentan síntomas significativos.

El cierre percutáneo es una alternativa a la cirugía en pacientes seleccionados, siendo una técnica segura y eficaz. La asociación de CIA y nacimiento anómalo de una de las arterias coronarias con recorrido retroaórtico es aún más infrecuente, adquiriendo relevancia clínica debido a que el borde anterosuperior del defecto septal se encuentra en estrecha relación con la arteria anómala.

Esta situación se plantea como posible contraindicación para cierre percutáneo debido al riesgo de comprometer a la arteria coronaria lo cual puede manifestarse con isquemia miocárdica, arritmia o muerte súbita.

\section{REPORTE DE UN CASO CLÍNICO}

Paciente masculino de 18 años y $90 \mathrm{~kg}$ de peso, sin antecedentes cardiovasculares personales y familiares. Sintomático por disnea en clase funcional I de 1 año de evolución. 


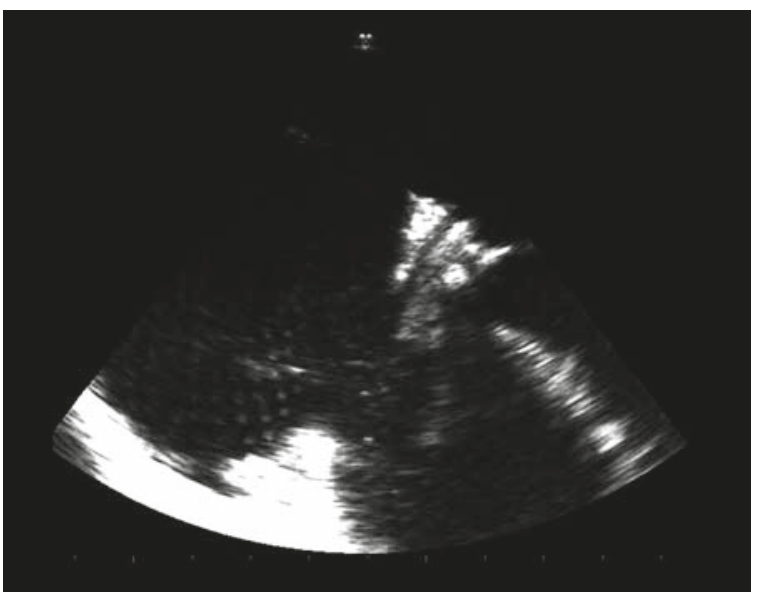

Figura 1. Imagen de ETE preprocedimiento. Corte medioesofágico a $0^{\circ}$ en vis ta de 4 cámaras focalizado en aurícula derecha y septum Interauricular. Se observa el defecto interauricular como asi también en su borde anterosuperior, una imagen hipoecoica lineal compatible con el recorrido retroaórtico de un vaso coronario (flecha blanca).

Exámen físico: R2 desdoblado fijo. Soplo sistólico 3/6 en foco pulmonar. Auscultación pulmonar normal. Pulsos periféricos conservados.

Electrocardiograma: Ritmo sinusal. Sobrecarga auricular derecha.

Teleradiografía de tórax: Leve aumento de la silueta cardíaca, con prominencia de la arteria pulmonar y leve redistribución de flujo.

Ecocardiograma Doppler color transtorácico (ECO) informa CIA tipo ostium secundum de $19 \times 18 \mathrm{~mm}$, dilatación leve de cavidades derechas y presión de arteria pulmonar normal, confirmado esto último por ecocardiograma transesofágico (ETE) el cual también observó bordes suficientes del defecto para intentar cierre percutáneo.

Bajo anestesia general, el ETE intraprocedimiento mostró una CIA de $22 \times 19 \mathrm{~mm}$ con bordes adecuados. Se detecta una anomalía no diagnosticada previamente. Se observa imagen hipoecoica con recorrido retroaórtico sugestivo de nacimiento anómalo de una arteria coronaria. (Figura 1)

Por este motivo se realiza coronariografía selectiva confirmando el nacimiento anómalo de la arteria circunfleja en el seno coronariano derecho con recorrido retroaórtico. (Figura 2)

Continuamos con las mediciones de la CIA para reevaluar su cierre percutáneo. Se avanzó balón elastomérico para medir el tamaño distendido de la CIA. Se posiciona y se insufla utilizando la técnica de $\bigotimes_{\text {stop- }}$ flow ${ }^{\circ}$ para medir la indentación en el mismo. El defecto midió $24.8 \mathrm{~mm}$ tanto por ETE como por radioscopia. Luego de esta valoración se decide continuar con el procedimiento.

Se posicionó vaina de Mullins (10F) en aurícula izquierda y se avanzó un dispositivo Amplatzer ${ }^{\circ}$ (St Jude-AGA Mn, USA) de $26 \mathrm{~mm}$ de cintura desplegando ambos discos en forma habitual. Luego de comprobar su correcta posición realizando test de

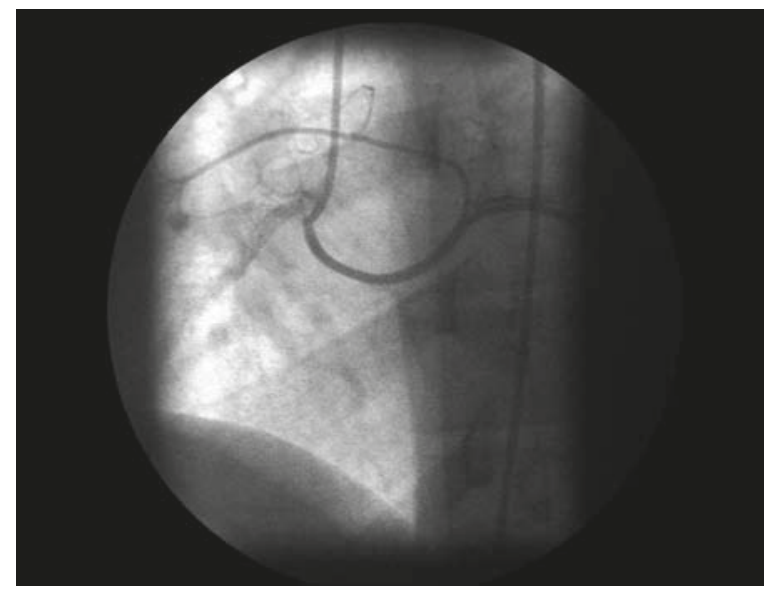

Figura 2. Angiografía coronaria selectiva en proyección oblicua anterior izquierda demostrando el origen anómalo de la arteria circunfleja originada en el seno aórtico derecho con recorrido retroaórtico.

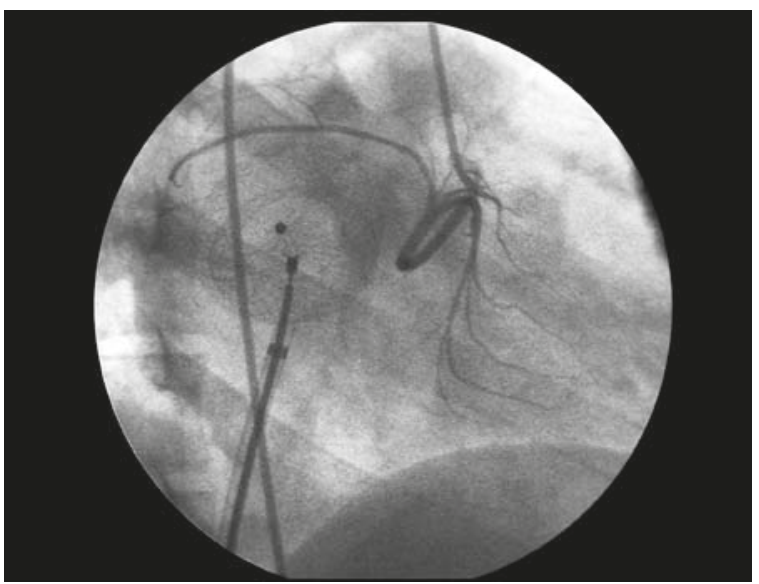

Figura 3. Angiografía coronaria selectiva en proyección oblicua anterior derecha. Se confirma la no afectación del recorrido de la arteria circunfleja previamente a la liberación del dispositivo oclusor.

estabilidad septal (maniobra de Minnesota), se realizó arteriografía selectiva de la arteria circunfleja corroborando que el dispositivo in situ no distorsionara ni comprimiera a esta última. (Figura 3). El monitoreo electrocardiográfico tampoco presentó cambios para isquemia, por lo que se procedió a liberar el dispositivo.

El paciente fue derivado a la Unidad Coronaria por 24 hs. Se le realizó ecocardiograma Doppler color transtorácico y teleradiografía de tórax antes del alta. Ambos estudios fueron normales.

A 3 y 6 meses de seguimiento se realizó un ecocardiogama Doppler color, que mostró la normoposición del dispositivo sin flujo residual y ECG/Holter que descartaron arritmias y signos de isquemia miocárdica.

A los 7 meses de se realizó una Tomografía Multislice (TAC MS) con el objetivo de demostrar la estrecha relación del dispositivo con la arteria coronaria circunfleja con recorrido retroaórtico. Se comprobó la correcta posición del dispositivo y la no compresión ni distorsión de la arteria circunfleja. (Figura 4) 


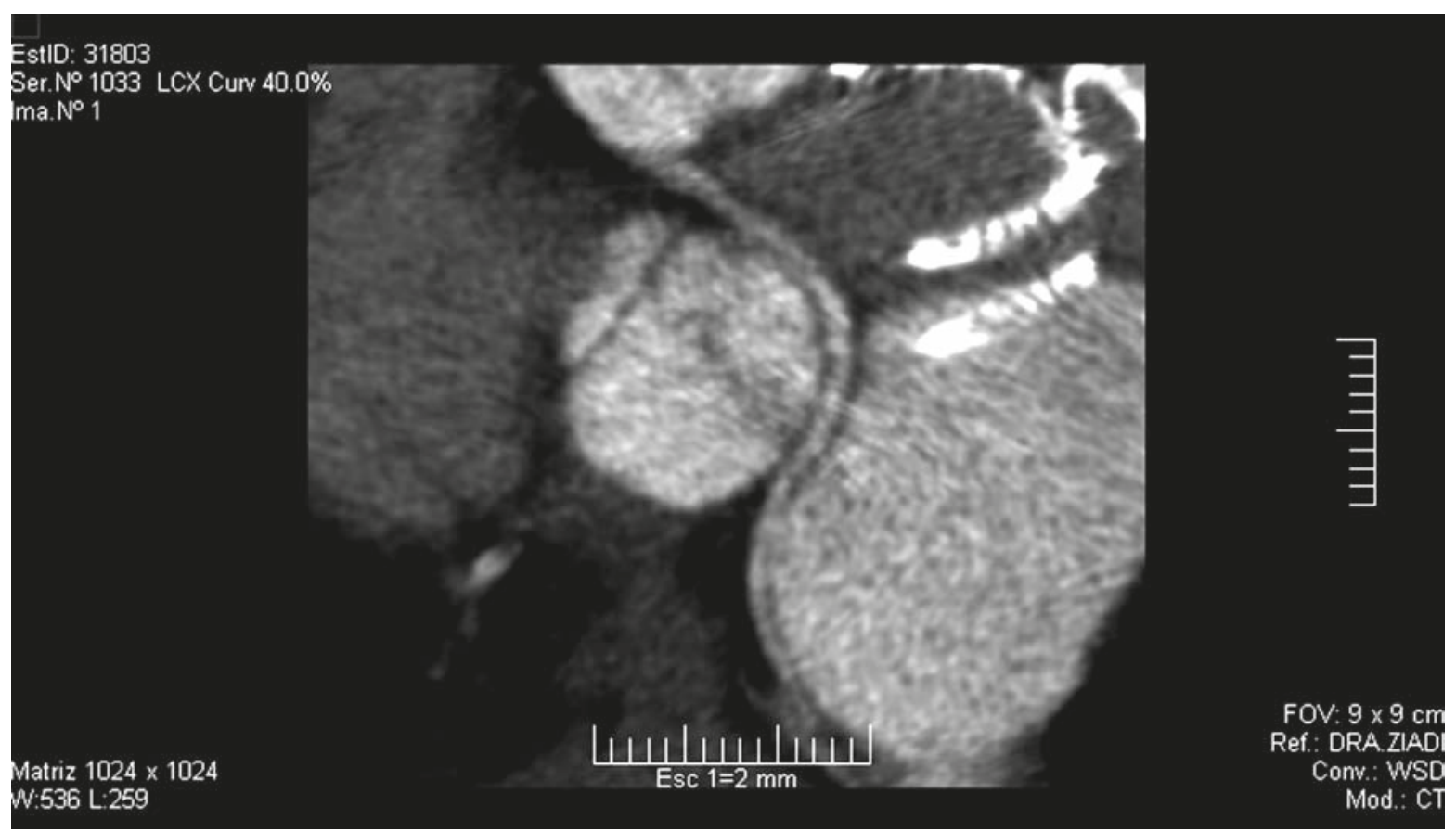

Figura 4. Imagen de TAC Multislice durante el seguimiento. Se observa la estrecha relación del dispositivo oclusor implantado (flecha negra) con la arteria circunfleja con recorrido retroaórtico descartándose compromiso en el recorrido de esta última (flecha blanca).

A los 14 meses se realiza una Prueba Ergométrica Graduada la cual informa buena capacidad funcional al máximo esfuerzo y sin alteraciones electrocadiográficas.

El paciente no presenta signos ni síntomas isquémicos a 24 meses de seguimiento post-procedimiento.

\section{DISCUSIÓN}

El tratamiento de la CIA es muchas veces motivo de discusión. El cierre percutáneo es el método de elección en 1) defectos de tipo Ostium Secundum, 2) diámetro menor o igual a $40 \mathrm{~mm}, 3) \mathrm{QP} / \mathrm{QS}$ igual o mayor a $1,5: 1,4)$ dilatación y sobrecarga de volumen de $\mathrm{VD}, 5)$ distancia de los márgenes mayor o igual a $5 \mathrm{~mm}$ (a seno coronario, válvula $\mathrm{AV}$, vena pulmonar superior derecha y aorta) y, 6) sospecha de embolia paradojal en pacientes con stroke criptogénico

Dentro de las contraindicaciones se mencionan: otra cardiopatía congénita que requiera intervención quirúrgica concomitante; CIA tipo Ostium Primum o Seno Venoso; peso menor a $8 \mathrm{~kg}$; procesos infecciosos locales o sistémicos; hipertensión arterial pulmonar con cortocircuito bidireccional o derecha izquier$\mathrm{da}$, o resistencia vascular pulmonar mayor a 7 unidades Woods.

El ETE tiene un rol fundamental ya que valora los bordes del defecto con estructuras vecinas (venas pulmonares, seno coronario, venas cavas, aorta, válvulas aurículo-ventriculares) para definir la conducta terapéutica, ya que si éstos son mayores a $5 \mathrm{~mm}$ el cierre percutáneo es recomendable. Durante el procedimiento de cierre percutáneo, el ETE demostrará la anatomía del defecto, descartará defectos múltiples y la distancia de separación entre los mismos, colaborará en la selección del dispositivo, controlará su posicionamiento durante y después de la liberación y finalmente descartará cortocircuitos residuales significativos.

Como se demuestra en este caso clínico, es esencial un cuidadoso y exhaustivo estudio ecocardiográfico en busca de anomalías asociadas a la CIA que deben ser tenidas en cuenta a la hora de decidir y llevar a cabo el cierre percutáneo.

El origen anómalo de la circunfleja en el seno derecho y con recorrido retroaórtico puede estar asociado a la CIA. El implante de un dispositivo oclusor puede provocar la compresión extrínseca y/o distorsión del vaso anómalo. Si una alteración anatómica o funcional del vaso coronario ocurriese luego del cierre percutáneo del defecto, puede manifestarse mediante la aparición de angina de pecho, síncope y arritmias hasta la ocurrencia muerte súbita.

En nuestro caso fue importante el hallazgo de la anomalía coronaria en el ETE intraprocedimiento y la confirmación angiográfica, previo al implante del dispositivo, ya que de no ser advertida, puede ser la causa de una severa complicación. Es mandatorio evaluar el borde anterosuperior del defecto y su relación con la arteria anómala con recorrido retroaórtico. Si el borde es suficiente, el dispositivo puede implantarse si no se observa compresión coronaria en la angiografía de control previa a su liberación. La angiografía post implante, un ECG normal durante el procedimiento y las imágenes del seguimiento con TAC MS, confirmaron la no afectación del vaso anómalo. Además la prueba de esfuerzo realizada fue negativa para isquemia. 


\section{CONCLUSIÓN}

El cierre percutáneo de una CIA tipo ostium secundum asociado al nacimiento anómalo de la arteria circunfleja con recorrido retroaórtico, fue eficaz y seguro realizando un detallado análisis mediante ETE, ECG y angiografía previa al implante del dispositivo.

\section{BIBLIOGRAFÍA}

1. Casolo G, Gensini GF, Santoro G, Rega L. Anomalous origin of the circunflex artery and patent foramen ovale: A rare cause of myocardial ischemia after percutaneous closure of the defect. Heart 2003; 89: e23.

2. Scholtz W, Jategaonkar S, Faber L, Horstkotte D. Unusual complication with transcatheter closure of an atrial septal defect prevented by adequate imaging. Circulation 2008; 117: e181-83.

3. Bijulal S, Krishnamoorthy K, Sivasankaran S. Retroaortic Coronary Artery:
Una valoración detallada de una CIA buscando anomalías asociadas nos permitió disminuir potenciales riesgos durante y posteriores al procedimiento.

El seguimiento alejado es importante para evaluar comportamiento del dispositivo dada su estrecha relación con un vaso coronario anómalo con recorrido retroaórtico.
Possible Contraindication for Device Closure of Atrial Septal Defect. Pediatr Cardiol 2011;32: 1001-03

4. Maki F, Ohtsuka T, Suzuky M, Hara Y, Shigematsu Y, Hamada M, Kawachi K, Hiwada K. Myocardial ischemia induced by anomalous aortic origin of the right coronary artery in a patient with atrial septal defect. Jpn Heart J 2001;42:371-6 